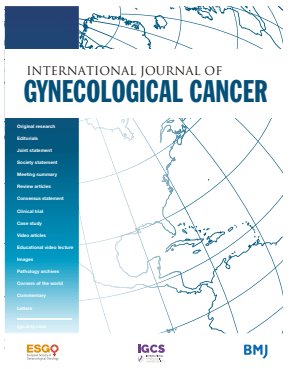

${ }^{1}$ Department of Obstetrics and Gynecology, Division of Gynecologic Oncology, University of Toronto, Toronto, Ontario, Canada

${ }^{2}$ Department of Surgical Oncology, Division of Gynecologic Oncology, University Health Network, Toronto, Ontario, Canada

Correspondence to Dr Taymaa May; taymaa.may@ uhn.ca

Accepted 12 July 2021 Published Online First 23 July 2021

\title{
High laparoscopic bilateral ovarian transposition to the upper abdomen prior to pelvic radiotherapy
}

Zibi Marchocki, ${ }^{1}$ Taymaa May ${ }^{1,2}$

Ovarian transposition offers preservation of hormonal function and fertility potential in women receiving pelvic radiation therapy for various malignancies (eg, cervical/colorectal cancer, pelvic lymphoma/ sarcoma). ${ }^{1}$ The procedure has been recommended for those with low risk of ovarian metastases and with acceptable ovarian reserve. ${ }^{2}$

The first ovarian transposition described by Batten and Brown in 1952 in a patient with pelvic neuroblastoma involved wrapping of the ovaries sutured together with a homemade lead sheath and attaching them to the anterior parietal peritoneum. ${ }^{3}$ Since then different surgical approaches have been described including open/laparoscopic approach, with different transposition sites such as the psoas muscles and the paracolic gutters, ${ }^{1}$ and with ${ }^{4}$ or without ${ }^{5}$ retroperitoneal tunneling of the ovarian vessels.

We describe a surgical technique of high ovarian transposition without retroperitoneal tunneling of ovarian vessels. First, the gonadal vessels are mobilized retroperitoneally toward their origin - from the aorta and inferior vena cava on the right and the aorta and left renal vein on the left. The ovaries are then lateralized to the upper abdomen without compromising the blood supply. This technique offers the advantage of minimizing the potential risk of ovarian damage from exposure to scattered radiation. In addition, transposition of the ovaries to the upper abdomen allows the radiation field extension cephalad without compromising the ovarian function. Similar to other

\section{High laparoscopic bilateral ovarian transposition to the upper abdomen prior to pelvic radiotherapy}

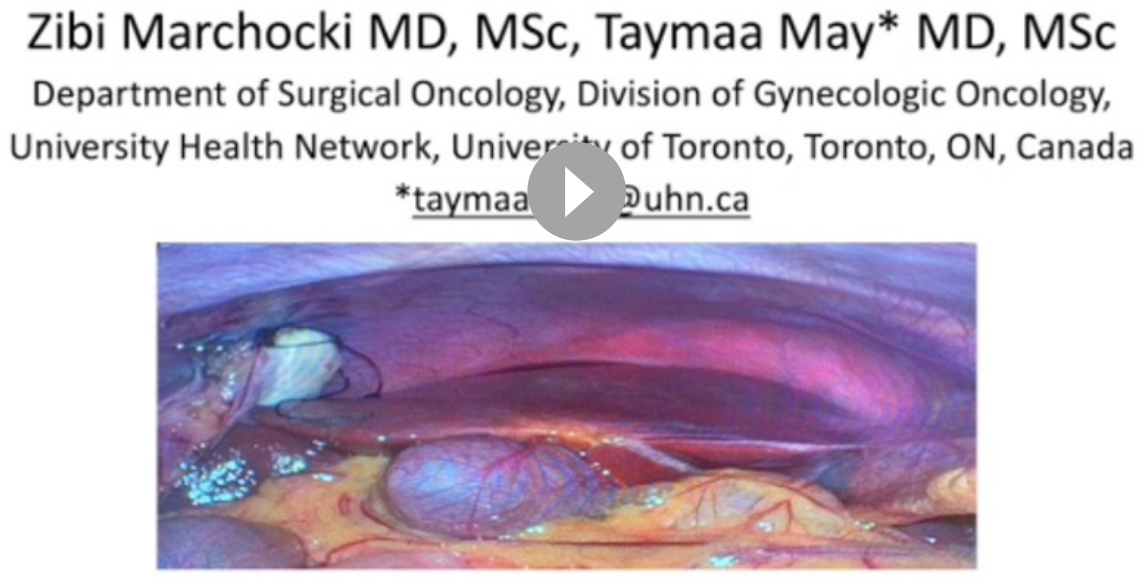

C) IGCS and ESGO 2021. No commercial re-use. See rights and permissions. Published by BMJ.

To cite: Marchocki Z, May T. Int J Gynecol Cancer 2021;31:1384-1385. 
Right
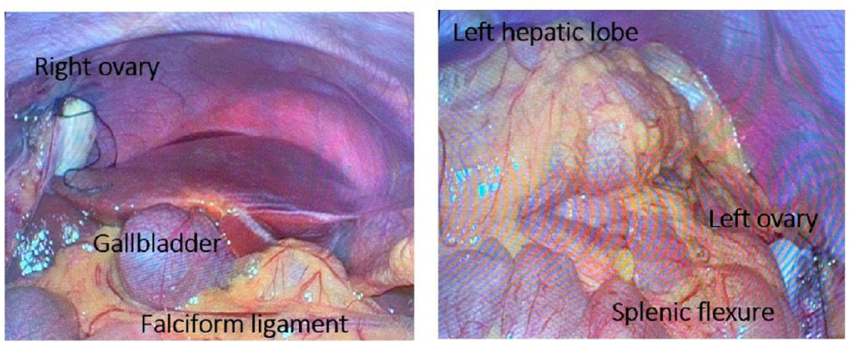

Figure 1 Transposed and secured ovaries above the inferior liver edge on the right and above the splenic flexure on the left.

reports, ${ }^{5}$ we had no incidences of bowel obstructions in our series of over 30 cases. The presented procedure was performed in a 19-year-old, GOPO woman with neurofibromatosis type 1 and $20 \mathrm{~cm}$ right groin malignant peripheral nerve sheath tumor awaiting pelvic radiotherapy. The appropriate patient consent has been obtained for the publication of this (Video 1).

The high laparoscopic ovarian transposition procedure includes: (1) inspection of the peritoneal cavity for metastases, (2) retroperitoneal dissection and creation of the perirectal and paravesical spaces, (3) bilateral resection of the utero-ovarian ligaments, (4) bilateral opportunistic salpingectomy, (5) complete bilateral ureterolysis (from the renal hilum to the pelvic brim), (6) retroperitoneal paraaortic dissection with bilateral ovarian vessels skeletonization to their origin inferior to the renal vessels, (7) bilateral colon mobilization to facilitate mobilization of the infundibulopelvic ligaments to the upper abdomen, (8) bilateral oophoropexy to the paracolic gutters - above the inferior liver edge on the right and above the splenic flexure on the left, while ensuring the blood supply is not twisted or kinked, (9) securing the ovaries to the peritoneum using permanent sutures such as 2.0 silk, and (10) application of titanium clips at the ovarian level for permanent radiation marking. Ovarian perfusion is assessed at the conclusion of the procedure by observing the patency of the gonadal vessels, the lack of torsion of the vascular pedicles, and the healthy color of the transposed ovaries. Long-term ovarian function can be assessed clinically using symptom assessments and biochemically using serum hormonal measurements.

Contributors ZM was responsible for conceptualization; video - image and video generation, montage, review, and editing; narration; writing - original draft; and writing - review and editing. TM was responsible for conceptualization; video - image and video generation, review and editing; writing - review and editing; supervision and copyright.

Funding The authors have not declared a specific grant for this research from any funding agency in the public, commercial or not-for-profit sectors.

Competing interests None declared.

Patient consent for publication Not required.

Provenance and peer review Not commissioned; externally peer reviewed.

Data availability statement All data relevant to the study are included in the article.

\section{REFERENCES}

1 Hoekman EJ, Broeders EABJ, Louwe LA, et al. Ovarian function after ovarian transposition and additional pelvic radiotherapy: a systematic review. Eur J Surg Oncol 2019;45:1328-40.

2 Anderson RA, Amant F, et al, ESHRE Guideline Group on Female Fertility Preservation. ESHRE guideline: female fertility preservation. Hum Reprod Open 2020;2020:hoaa052.

3 Batten R, Brown DEM. Protection of ovaries from radiation. Lancet 1956;270:939-40.

4 Arian SE, Goodman L, Flyckt RL, et al. Ovarian transposition: a surgical option for fertility preservation. Fertil Steril 2017;107:e15.

5 Huang K-G, Lee C-L, Tsai C-S, et al. A new approach for laparoscopic ovarian transposition before pelvic irradiation. Gynecol Oncol 2007;105:234-7 